Author affiliations and support information (if applicable) appear at the end of this article.

Published at jco.org on May 24, 2018.

A.T.B., N.C.T.v.G., and B.Y. contributed equally to this work.

Clinical trial information: NCT02271464

Corresponding author: Bauke Ylstra, PhD, Department of Pathology, VU University Medical Center, PO Box 7057, 1007 MB Amsterdam, the Netherlands; e-mail: b. ylstra@vumc.nl.

(C) 2018 by American Society of Clinical Oncology

$0732-183 X / 18 / 3620 w-2052 w / \$ 20.00$

\title{
Loss of Chromosome 18q11.2-q12.1 Is Predictive for Survival in Patients With Metastatic Colorectal Cancer Treated With Bevacizumab
}

\begin{abstract}
Erik van Dijk, Hedde D. Biesma, Martijn Cordes, Dominiek Smeets, Maarten Neerincx, Sudipto Das, Paul P. Eijk, Verena Murphy, Anna Barat, Orna Bacon, Jochen H.M. Prehn, Johannes Betge, Timo Gaiser, Bozena Fender, Gerrit A. Meijer, Deborah A. McNamara, Rut Klinger, Miriam Koopman, Matthias P.A. Ebert, Elaine W. Kay, Bryan T. Hennessey, Henk M.W. Verheul, William M. Gallagher, Darran P. O'Connor, Cornelis J.A. Punt, Fotios Loupakis, Diether Lambrechts, Annette T. Byrne, Nicole C.T. van Grieken, and Bauke Ylstra
\end{abstract}

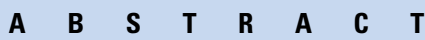

\section{Purpose}

Patients with metastatic colorectal cancer (mCRC) have limited benefit from the addition of bevacizumab to standard chemotherapy. However, a subset probably benefits substantially, highlighting an unmet clinical need for a biomarker of response to bevacizumab. Previously, we demonstrated that losses of chromosomes 5q34, 17q12, and 18q11.2-q12.1 had a significant correlation with progression-free survival (PFS) in patients with $\mathrm{mCRC}$ treated with bevacizumab in the CAIRO2 clinical trial but not in patients who did not receive bevacizumab in the CAIRO trial. This study was designed to validate these findings.

\section{Materials and Methods}

Primary $\mathrm{mCRC}$ samples were analyzed from two cohorts of patients who received bevacizumab as first-line treatment; 96 samples from the European multicenter study Angiopredict (APD) and 81 samples from the Italian multicenter study, MOMA. A third cohort of 90 samples from patients with $\mathrm{mCRC}$ who did not receive bevacizumab was analyzed. Copy number aberrations of tumor biopsy specimens were measured by shallow whole-genome sequencing and were correlated with PFS, overall survival (OS), and response.

\section{Results}

Loss of chromosome 18q11.2-q12.1 was associated with prolonged PFS most significantly in both the cohorts that received bevacizumab (APD: hazard ratio, 0.54; $P=.01$; PFS difference, 65 days; MOMA: hazard ratio, 0.55; $P=.019 ;$ PFS difference, 49 days). A similar association was found for OS and overall response rate in these two cohorts, which became significant when combined with the CAIRO2 cohort. Median PFS in the cohort of patients with $\mathrm{mCRC}$ who did not receive bevacizumab and in the CAIRO cohort was similar to that of the APD, MOMA, and CAIRO2 patients without an 18q11.2-q12.1 loss.

\section{Conclusion}

We conclude that the loss of chromosome 18q11.2-q12.1 is consistently predictive for prolonged PFS in patients receiving bevacizumab. The predictive value of this loss is substantiated by a significant gain in OS and overall response rate.

\section{J Clin Oncol 36:2052-2060. (C) 2018 by American Society of Clinical Oncology}

\section{INTRODUCTION}

Standard chemotherapeutic first-line treatment of patients with metastatic colorectal cancer (mCRC) consists of a fluoropyrimidine combined with either irinotecan or oxaliplatin and a targeted agent. Targeted agents are either cetuximab or panitumumab, which are both epidermal growth factor receptor (EGFR) inhibitors, or bevacizumab, a vascular endothelial growth factor (VEGF) inhibitor. ${ }^{1}$ The addition of bevacizumab to first-line combination chemotherapy in two large randomized phase III mCRC clinical trials, by Hurwitz et $\mathrm{al}^{2}$ and Saltz et $\mathrm{al}^{3}$ showed inconsistent results on median progression-free survival (PFS) and overall survival (OS). ${ }^{2,3}$ Two explanations for this inconsistency have been 
presented: (1) the use of a regimen with bolus fluorouracil (FU) plus irinotecan in Hurwitz et $\mathrm{al}^{2}{ }^{2}$ which, because of its toxicity, is now considered obsolete; and (2) the inconsistent treatment continuation until progressive disease in Saltz et al. ${ }^{3}$ The importance of bevacizumab continuation was demonstrated recently by the results of the CAIRO3 trial. ${ }^{4}$ The benefit of bevacizumab, however, is associated with a high cost and an appreciable toxicity profile. $^{2,5-7}$ Consequently, and despite international guidelines, ${ }^{1}$ bevacizumab is not available in all countries. ${ }^{8}$ A predictive biomarker for bevacizumab is thus an urgent clinical need, as acknowledged by the European Society for Medical Oncology. ${ }^{1}$

Several candidate biomarkers for bevacizumab, including tumor VEGF isoforms (VEGF-A, VEGF-B, VEGF-C, and VEGFD) and neuropilin-1, a coreceptor for VEGF, have been proposed. These markers have yielded promising results in small exploratory studies, but validation in larger independent trials has failed. ${ }^{9-11}$

In a genome-wide screen of 119 well-annotated mCRC tumors from patients treated with bevacizumab from the CAIRO2 phase III clinical trial, ${ }^{12}$ losses at chromosomes $5 \mathrm{q}, 17 \mathrm{q}$, and $18 \mathrm{q}$ correlated significantly with prolonged PFS, with the highest significance at the chromosomal regions 18q11.2-q12.1, 5q34, and $17 \mathrm{q} 12 .{ }^{13}$ Although the strongest trend was observed for region 18q11.2-q12.1, almost all patients with a loss of region 18q11.2q12.1 lost the entire chromosomal arm 18q, rendering this finding comparable to that of previous publications in which loss of chromosomal arm 18q was found to be a prognostic marker. ${ }^{14-17}$ In 186 patients from the CAIRO phase III clinical trial, ${ }^{18}$ in which patients received sequential or combination chemotherapy but no bevacizumab, the same chromosomal regions did not correlate with PFS. ${ }^{13}$ It could not be concluded whether the biomarker candidates would be predictive for oxaliplatin or bevacizumab, because the CAIRO2 patients had received bevacizumab combined with capecitabine and oxaliplatin, whereas the CAIRO patients had received either capecitabine or capecitabine and irinotecan but neither bevacizumab nor oxaliplatin. Here we present validation data from three additional multicenter cohorts showing that chromosomal region 18q11.2-q12.1 has predictive value for bevacizumab in mCRC.

\section{MATERIALS AND METHODS}

\section{Patient Samples}

Samples from patients with histologically proven mCRC or locally irresectable colorectal cancer treated with standard first-line combination chemotherapy with or without bevacizumab with extensive clinical followup were included in this study.

Formalin-fixed and paraffin-embedded primary tumor samples from three cohorts were assembled for clinical validation purposes: (1) the Angiopredict (APD) cohort (96 tumor samples from patients receiving chemotherapy supplemented with bevacizumab in first-line treatment $)^{19}$; (2) the Maintenance Bevacizumab Only or Bevacizumab Plus Metronomic Chemotherapy in Advanced Colorectal Cancer (MOMA) cohort (81 tumor samples from the MOMA clinical trial of patients receiving chemotherapy supplemented with bevacizumab in first-line treatment [ClinicalTrials.gov identifier: NCT02271464] $)^{20}$; and (3) the NoBev cohort (90 tumor samples from patients who did not receive bevacizumab and received standard combination chemotherapy regimens similar to that of the APD cohort ${ }^{19}$; Table 1). Of the 90 NoBev samples, 80 are also included in Neerincx et al (manuscript in preparation) and 38 of those are included in Smeets et al (manuscript in preparation). Tand $\mathrm{N}$ classification, grading, and tumor location were collected by reviewing patient records. $\mathrm{T}$ and $\mathrm{N}$ classifications and grading were evaluated routinely by pathologists from the participating centers using the American Joint Committee Classification 7 th edition cancer staging manual classification. ${ }^{21}$ TNM staging and age were not significantly correlated with bevacizumab supplementation in either the APD or the NoBev cohort (Table 2).

Chemotherapy in the APD and NoBev cohorts was variable yet balanced and consisted of capecitabine or FU and leucovorin combined with either oxaliplatin, irinotecan, or both (Table 1). Combination chemotherapy in the MOMA cohort was FU and leucovorin, oxaliplatin, and irinotecan supplemented with bevacizumab in first-line treatment.

Array comparative genomic hybridization (arrayCGH) copy number data from the CAIRO and CAIRO2 cohorts were retrieved from the gene expression omnibus (data set GSE3684). Samples from patients receiving fewer than three cycles of chemotherapy and samples with microsatellite instability (MSI) were excluded by Haan et al. ${ }^{13}$ However, these data were included here to render the CAIRO and CAIRO2 cohorts more comparable to the APD and NoBev cohorts, in which MSI status and the number of cycles to progression were not considered ${ }^{19}$ because they were not always available. This added arrayCGH data from a total of 44 samples to the CAIRO and CAIRO2 data sets. In the CAIRO cohort, seven samples were MSI positive ${ }^{22}$ and 24 patients received fewer than three cycles of chemotherapy (total expanded data set $[\mathrm{n}=216$ samples]). In the CAIRO2 cohort, 12 samples were MSI positive ${ }^{22}$ and two patients received fewer than three cycles of chemotherapy (total expanded data set $[\mathrm{n}=133$ samples $]$ ).

The conclusions by Haan et $\mathrm{al}^{13}$ remained unaffected after expansion of the data set (Fig 1A). The overall median PFS in the CAIRO2 cohort was 326 days in Haan et al, ${ }^{13}$ and it became 323 days in the expanded data set. Significance for $5 \mathrm{q} 34$ remained unchanged and was reduced from 0.004 to 0.045 for $17 \mathrm{q} 12$. In the CAIRO cohort, median PFS was 224 days in Haan et $\mathrm{al},{ }^{13}$ and PFS was 249 days in the expanded data set.

For technical validation, arrayCGH results by Haan et $\mathrm{al}^{13}$ were compared with shallow next generation sequencing (NGS) results. Therefore, DNA previously isolated and used for the arrayCGH experiments ${ }^{13}$ from 96 tumor samples from the CAIRO2 trial were subjected to shallow NGS.

\section{Power Calculation and Statistics}

Power analysis was performed using the proportional hazards cure model. ${ }^{23}$ Data from the CAIRO2 trial $^{13}$ were used to estimate the hazard

Table 1. Chemotherapy Regimens for All Three Validation Cohorts and the CAIRO and CAIRO2 Cohorts of the Extended Data Set

\begin{tabular}{lccccc}
\hline \multicolumn{1}{c}{ Cohort } & APD & NoBev & MOMA & CAIRO & CAIRO2 \\
\hline CAPOX/FOLFOX & $56(58)$ & $45(50)$ & $0(0)$ & $0(0)$ & $133(100)$ \\
CAPIRI/FOLFIRI & $30(31)$ & $16(18)$ & $0(0)$ & $111(51)$ & $0(0)$ \\
CAP/FU* & $5(5)$ & $17(19)$ & $0(0)$ & $105(49)$ & $0(0)$ \\
FOLFOXIRI & $0(0)$ & $3(3)$ & $81(100)$ & $0(0)$ & $0(0)$ \\
Othert & $5(5)$ & $9(10)$ & $0(0)$ & $0(0)$ & $0(0)$ \\
Total & $96(100)$ & $90(100)$ & $81(100)$ & $216(100)$ & $133(100)$
\end{tabular}

NOTE. Data are presented as No. (\%)

Abbreviations: APD, Angiopredict; CAP/FU, capecitabine or fluorouracil alone CAPIRI/FOLFIRI, capecitabine or fluorouracil in combination with irinotecan; CAPOX/FOLFOX, capecitabine or fluorouracil in combination with oxaliplatin: FOLFOXIRI, fluorouracil in combination with oxaliplatin and irinotecan; NoBev, cohort of patients who did not receive bevacizumab and received standard combination chemotherapy regimens similar to that of the APD cohort.

* Because bevacizumab is typically given in addition to combination chemotherapy, the group receiving CAP/FU monotherapy is slightly underrepresented in the APD cohort compared with the NoBev cohort ( $\chi^{2}$ test, $P=$ $.007)$

tOther refers to patients receiving some treatment in the Table supplemented by mitomycin $(n=1)$, panitumumab $(n=2)$, cetuximab $(n=3)$, aflibercept $(n=1)$, imatinib $(n=1)$, or raltitrexed $(n=1)$, monotherapy irinotecan $(n=2)$, or monotherapy bevacizumab $(n=3)$. 


\begin{tabular}{|c|c|c|c|c|}
\hline Cohort & APD & NoBev & $P$ & MOMA \\
\hline Age, years & 61.6 & 61.1 & .40 & 61 \\
\hline \multicolumn{5}{|l|}{ Sex } \\
\hline Male & $68(71)$ & $58(64)$ & \multirow[t]{4}{*}{.20} & $49(61)$ \\
\hline Female & $28(29)$ & $32(36)$ & & 31 (38) \\
\hline Not available & 0 & 0 & & $1(1)$ \\
\hline Total patients & $96(100)$ & $90(100)$ & & $81(100)$ \\
\hline \multicolumn{5}{|l|}{ T stage } \\
\hline $\mathrm{T} 1$ & $1(1)$ & $2(2)$ & \multirow[t]{5}{*}{.43} & $0(0)$ \\
\hline T2 & $11(11)$ & $6(7)$ & & $0(0)$ \\
\hline T3 & $61(64)$ & $50(56)$ & & $0(0)$ \\
\hline T4 & $22(23)$ & $29(32)$ & & $0(0)$ \\
\hline Not available & $5(5)$ & $3(3)$ & & $81(100)$ \\
\hline \multicolumn{5}{|l|}{$N$ stage } \\
\hline NO & $24(25)$ & $24(27)$ & \multirow[t]{4}{*}{.67} & $0(0)$ \\
\hline N1 & $28(29)$ & $29(32)$ & & $0(0)$ \\
\hline N2 & 37 (39) & $34(38)$ & & $0(0)$ \\
\hline Not available & 7 (7) & $3(3)$ & & $81(100)$ \\
\hline \multicolumn{5}{|l|}{ M stage } \\
\hline Mo & $34(35)$ & $38(42)$ & \multirow[t]{3}{*}{.42} & $0(0)$ \\
\hline M1 & $61(54)$ & $52(58)$ & & $0(0)$ \\
\hline Not available & $1(1)$ & $0(0)$ & & $81(100)$ \\
\hline \multicolumn{5}{|l|}{ Metastasis } \\
\hline To one organ & $0(0)$ & $0(0)$ & \multirow[t]{3}{*}{-} & $34(42)$ \\
\hline To multiple organs & $0(0)$ & $0(0)$ & & $47(58)$ \\
\hline Not available & $96(100)$ & $90(100)$ & & $0(0)$ \\
\hline \multicolumn{5}{|l|}{ Adjuvant treatment } \\
\hline Received & $0(0)$ & $0(0)$ & \multirow[t]{3}{*}{-} & $10(12)$ \\
\hline Did not receive & $0(0)$ & $0(0)$ & & $71(88)$ \\
\hline Not available & $96(100)$ & $90(100)$ & & $0(0)$ \\
\hline
\end{tabular}

NOTE. Data are presented as No. (\%) unless indicated otherwise. M0 corresponds to patients with metachronous cancer, and M1 corresponds to patients with synchronous cancer. A $\chi^{2}$ test was used to calculate $P$ values for an association between the clinical variables and the addition of bevacizumab to chemotherapy between the APD and NoBev cohorts. No significant associations were found.

Abbreviations: APD, Angiopredict: NoBev, cohort of patients who did not receive bevacizumab and received standard combination chemotherapy regimens similar to that of the APD cohort.

ratio for patients with and without a loss, and to estimate the proportion of patients with a chromosomal aberration. For all three candidate biomarkers, loss of chromosomal regions $5 \mathrm{q} 34,17 \mathrm{q} 12$, and 18q11.2-q12.1, the minimal sample size was calculated for $\alpha=.05$ and $80 \%$ power. This demonstrated that a minimum number of 69 patient samples for the 18q11.2-q12.1 loss, 115 for the $5 \mathrm{q} 34$ loss, and 339 for the $17 \mathrm{q} 12$ loss was required to validate the results obtained by Haan et al. ${ }^{13}$ All cohorts were thus sufficiently powered to validate the 18q11.2-q12.1 loss. However, none of the cohorts was sufficiently powered to validate either the $5 q 34$ or the $17 \mathrm{q} 12$ losses; these losses were not considered for additional analysis.

Using the statistical programming language $\mathrm{R}$ ( $\mathrm{v}$ 3.4.0) and package CGHtest (v 1.1), a two-sided log-rank test was used to calculate a $P$ value for the association between copy number aberrations and PFS. ${ }^{24} \mathrm{~A} P$ value threshold of .05 was used to test the significance of the association between 18q11.2-q12.1 loss and PFS. In these cohorts, only chromosome 18q11.2- q12.1 loss was tested; hence, no multiple testing correction was applied. The interaction $P$ value was calculated for the interaction of PFS with bevacizumab. OS and the overall response rate (ORR) were evaluated as secondary end points.

Correlations between loss of 18q11.2-q12.1 and standard prognostic factors serum lactate dehydrogenase elevation (LDH; normal $v$ abnormal), age, sex, number of affected organs (one $v$ more), and previously received adjuvant treatment were investigated for the CAIRO and CAIRO2 cohorts. These correlations were not calculated by Haan et al. ${ }^{13}$ Equivalent calculations were not possible for the APD and NoBev cohorts because the number of affected organs, previously received adjuvant treatment, and LDH status were not available. For the MOMA trial, this calculation was performed for age, sex, number of affected organs and previously received treatment, but not for LDH status because that status was not available.

\section{Sequencing and Processing}

Copy number profiles of samples from all three validation cohorts and the 96 matched CAIRO2 samples were obtained for technical validation using shallow NGS as described by Scheinin et al. ${ }^{25}$ Details are summarized in the Data Supplement. Algorithms and settings to call chromosomal copy numbers gains and losses and to determine chromosomal regions were performed according to Haan et al ${ }^{13,26-29}$ Shallow NGS data have been deposited at the European Genome-phenome Archive (EGA; http://www.ebi.ac.uk/ega/), which is hosted by the European Bioinformatics Institute, under accession numbers EGAS00001002724 (APD and NoBev cohorts) and EGAS00001002617 (MOMA cohort and CAIRO2 technical validation samples).

\section{RESULTS}

\section{Comparison Between Next-Generation Sequencing and arrayCGH}

Technical validation of the shallow NGS procedures for copy number analysis was performed for 96 of the CAIRO2 samples analyzed previously by arrayCGH in Haan et al. ${ }^{13}$

Frequency plots for arrayCGH and shallow NGS copy number aberrations were comparable (Data Supplement). Genome-wide, the 30-kbp bins used in our shallow NGS analysis that are covered by an arrayCGH probe showed a $92 \%$ concordance in copy number calling, which once again technically validates the copy number results obtained by shallow NGS compared with arrayCGH. ${ }^{25}$ For the 18q11.2-q12.1 region, 91 of the 96 samples have the same copy number calls. Despite these five discordant calls, survival analysis for the 96 samples by shallow NGS shows that the conclusions remained the same and are in concordance with the arrayCGH results (Data Supplement). ${ }^{25,30}$

\section{Landscape of Chromosomal Aberrations in the Different mCRC Cohorts}

We performed shallow NGS for 96 tumor samples from the APD cohort, 90 from the NoBev cohort, and 81 from the MOMA cohort. The frequency distribution of chromosomal gains and losses was similar for all three cohorts and was comparable with the frequency distributions presented previously for the CAIRO and CAIRO2 cohorts $^{13}$ (Data Supplement) and with the mCRC copy number data from The Cancer Genome Atlas. ${ }^{17}$

\section{Association With Survival}

Losses at three chromosomal regions, 5q34, 17q12, and 18q11.2-q12.1, were found previously to be the most significantly associated with PFS in the CAIRO2 treatment arm that was supplemented with bevacizumab ${ }^{13}$; these associations were not observed in the CAIRO data set. Given the power requirements, only chromosomal region 18q11.2-q12.1 could be tested in the APD, NoBev, and MOMA cohorts. However, these cohorts were too small to validate the $5 \mathrm{q} 34$ and $17 \mathrm{q} 12$ losses, because these copy number aberrations occur less frequently (Data Supplement) and would require cohorts of $n=115$ and $n=339$ or larger, respectively. A log-rank test was performed to determine the 


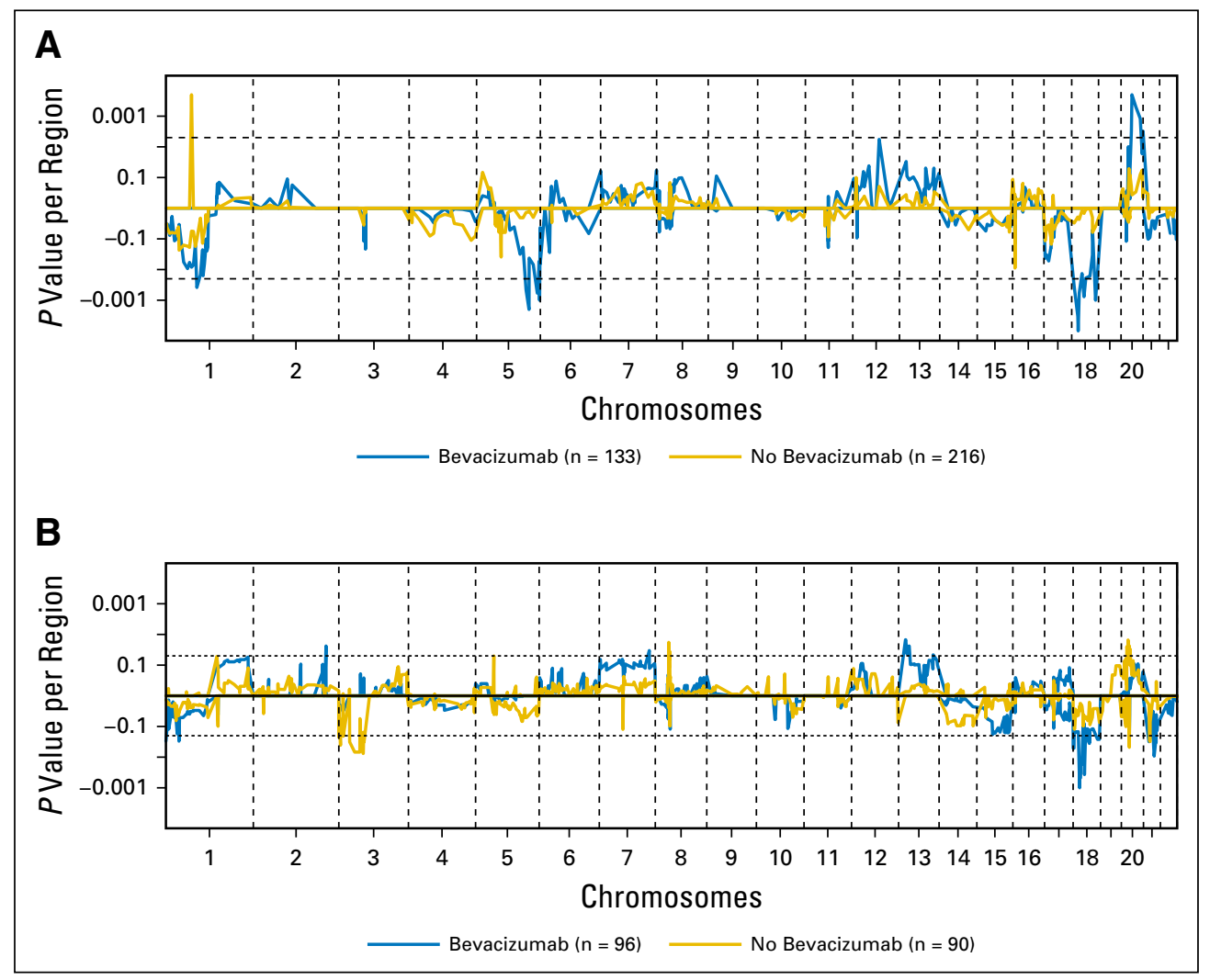

Fig 1. Genome-wide association of progression-free survival with either gain or loss in four cohorts. (A) CApecitabine, IRinotecan, and Oxaliplatin in advanced colorectal cancer (CAIRO) and CAIRO2 (extended) cohorts. (B) Angiopredict cohort (APD) and NoBev cohort (patients who did not receive bevacizumab and received standard combination chemotherapy regimens similar to that of the APD cohort). $P$ values of gains (positive values) or losses (negative values) are shown as a function of chromosomal regions sorted by chromosomal position ( $x$-axis). Vertical dotted lines show the chromosomal borders. Horizontal dotted lines show the significance thresholds $(P=.005$ for the CAIRO cohorts and $P=.05$ for the APD and NoBev cohorts). In both cohorts with bevacizumab treatment (CAIRO2 and APD, blue lines), the chromosomal region in $18 \mathrm{q}$ is significantly associated with survival. In both cohorts treated without bevacizumab (CAIRO and NoBev, gold lines), there is no significant association. Even though only chromosomal region 18q11.2-q12.1 has a $P$ value below the significance threshold, the same trend is observed for the entire chromosomal arm 18q for all cohorts treated with bevacizumab.

significance of associations between chromosome 18q11.2-q12.1 loss and PFS. This loss presented with a significant association with PFS for both patient cohorts receiving combination chemotherapy supplemented with bevacizumab: the APD cohort of 96 patients $(P=.01$; hazard ratio $[\mathrm{HR}], 0.54$; median PFS, 290 days with loss and 226 days without loss) and the MOMA cohort of 81 patients ( $P=$ .019 ; HR, 0.55; median PFS, 275 days with loss and 231 days without loss), consistent with the 133 patients included in the expanded CAIRO2 cohort $(P=.0086$; HR, 0.57; median PFS, 330 days with loss and 196 days without loss; Figs 2A-2C).

No significant association was found for the 90 patients in the NoBev cohort who received combination chemotherapy treatment without bevacizumab $(P=.47$; HR, 0.85 ; median PFS, 206 days with loss and 206 days without loss), consistent with the 216 patients included in the CAIRO cohort $(P=.82 ; \mathrm{HR}, 0.96$; median PFS, 225 days with loss and 192 days without loss; Figs 2A and $2 \mathrm{~B})$.

A combined analysis of all cohorts shows that chromosome 18q11.2-q12.1 loss provides an increase in median PFS of 109 days for patients treated with bevacizumab, when compared with patients without that loss or with patients who did not receive bevacizumab supplementation (Fig 2D).

The same analysis was performed for OS using a log-rank test to determine the significance of an association with chromosome 18q11.2-q12.1 loss. Of course, to calculate the association with OS, the heterogeneity of treatment modalities in second and subsequent treatment lines could not be considered, and this is a confounding factor. Nevertheless, for all cohorts, OS showed a trend similar to that of PFS. For the APD and MOMA cohorts, these trends were not statistically significant, but in a combined analysis that included the CAIRO2 cohort, a significant association was observed $(P=.039$; HR, 0.73; median OS, 584 days with loss and 465 days without loss; Fig 3). No significant association between OS and 18q11.2-q12.1 loss was found for either the NoBev or the CAIRO cohorts, either alone or combined (Fig 3).

ORR was available for 566 of 616 patients; it was higher in patients with a loss of chromosomal region 18q11.2-q12.1 in all three cohorts treated with bevacizumab, consistent with the results for PFS and OS. This association was statistically significant in the CAIRO2 cohort, but not in the MOMA or APD cohort, both of which suffer from lower patient numbers. In a combined analysis, a significant association was found for patients treated with bevacizumab $(P=.0033)$ but not for patients who did not receive bevacizumab $(P=.20$; Data Supplement $)$.

\section{Association of 18q11.2-q12.1 Loss With Known Prognostic Variables}

In a combined analysis of the CAIRO and CAIRO2 cohorts, ${ }^{12,18}$ the number of affected organs and $\mathrm{LDH}$ status were significant prognostic factors. In the CAIRO cohort, in a multivariate Cox model, loss of 18q11.2-q12.1 was not significantly associated with PFS. In the CAIRO2 cohort, in a multivariate Cox model, loss of 18q11.2-q12.1 remained significantly associated with PFS. In the MOMA cohort, LDH status was not available. In a multivariable Cox model, loss of 18q11.2-q12.1 remained significantly associated with PFS (Data Supplement). 


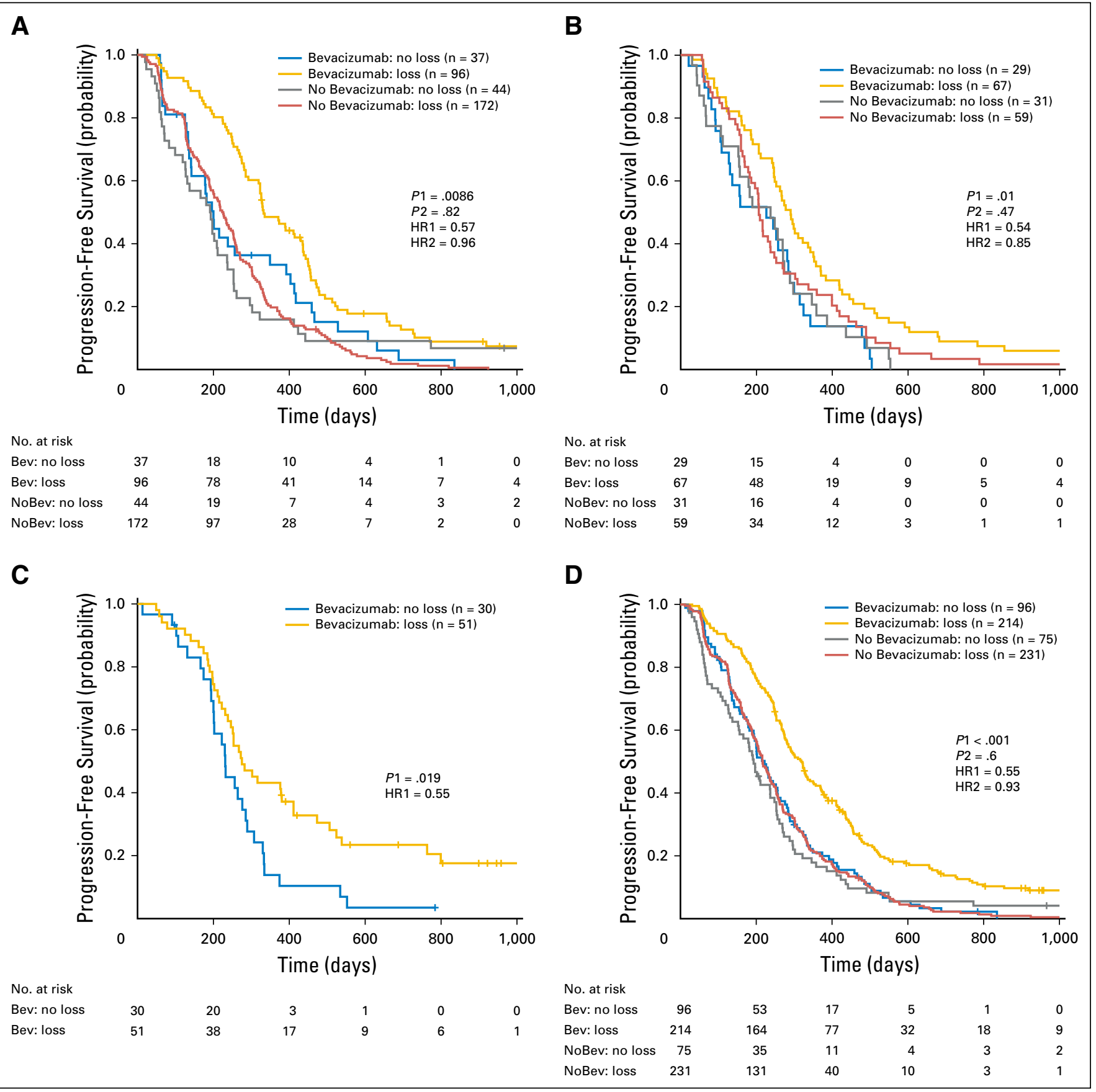

Fig 2. Kaplan-Meier survival analysis for loss versus no loss of 18q11.2-q12.1 with respect to progression-free survival for all cohorts, and a meta-analysis of the combined

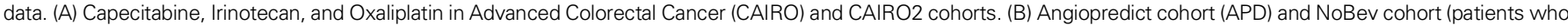

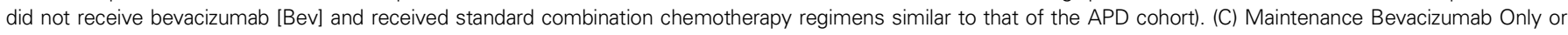

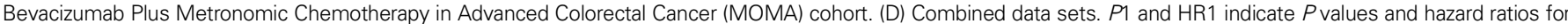
patients treated with Bev (blue lines $v$ gold lines). $P 2$ and HR2 indicate significance and hazard ratios for patients treated without Bev (red lines $v$ gray lines).

For the clinical variables available, the same analysis was performed in the APD and NoBev cohorts. There was no significant bias for age $(P=.40)$, sex $(P=.20)$, T stage $(P=.43)$, $\mathrm{N}$ stage $(P=.67)$, or $\mathrm{M}$ stage $(P=.42)$ in the selection criteria for the APD versus the NoBev cohort (Table 2). Loss of chromosomal region 18q11.2-q12.1 was also compared with standard prognostic factors. Again, no significant bias for sex $(P=.16)$, T stage $(P=.08)$, N stage $(P=.79)$, or $\mathrm{M}$ stage $(P=.22)$ was found (Table 3$)$. No multivariable Cox model was used for this cohort, because there were no significant associations between clinical variables and PFS (Data Supplement).

The interaction $P$ value of response to bevacizumab $\left(P_{\text {int }}\right)$ with respect to loss of chromosomal region 18q11.2-q12.1 was not significant for the expanded CAIRO and CAIRO2 cohort pair $\left(P_{\text {int }}=.06\right)$ or for the APD and NoBev cohort pair $\left(P_{\text {int }}=.14\right)$. Both cohort pairs were underpowered to detect an interaction $P$ value. Taken across all five cohorts, $P_{\text {int }}$ was .006 . We could not consider 


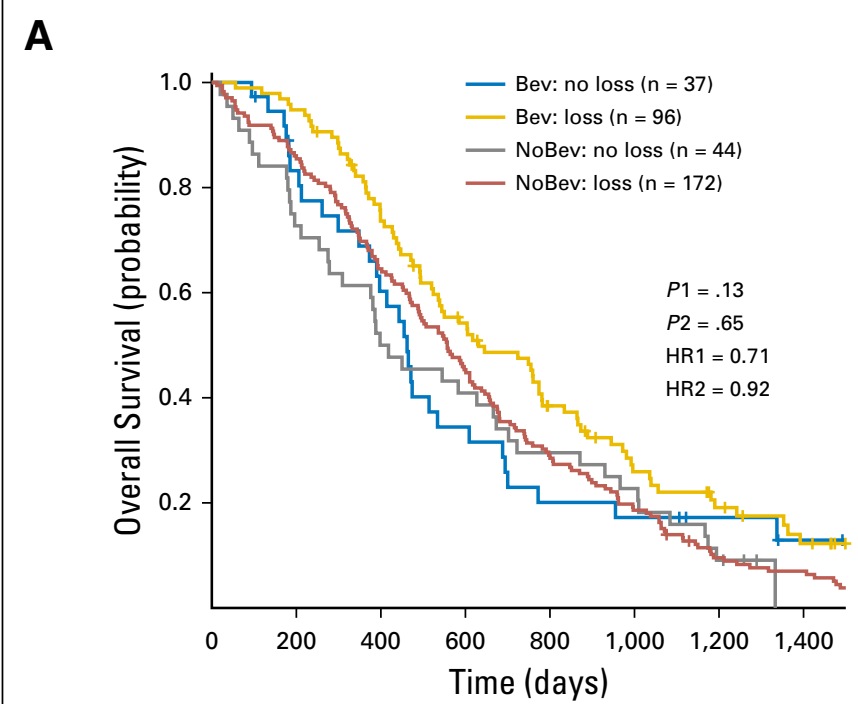

\section{B}

$\begin{array}{lrrrrrrrr}\text { No. at risk } & & & & & & & & \\ \text { Bev: no loss } & 37 & 29 & 21 & 12 & 7 & 6 & 4 & 2 \\ \text { Bev: loss } & 96 & 91 & 69 & 49 & 32 & 20 & 13 & 7 \\ \text { NoBev: no loss } & 44 & 32 & 22 & 18 & 13 & 10 & 4 & 0 \\ \text { NoBev: loss } & 172 & 148 & 111 & 78 & 50 & 32 & 15 & 11\end{array}$

$\begin{array}{llllrrrrr}\text { No. at risk } & & & & & & & \\ \text { Bev: no loss } & 29 & 22 & 18 & 10 & 8 & 7 & 5 & 3 \\ \text { Bev: loss } & 67 & 60 & 44 & 35 & 26 & 17 & 12 & 9 \\ \text { NoBev: no loss } & 31 & 24 & 17 & 10 & 6 & 3 & 0 & 0 \\ \text { NoBev: loss } & 59 & 49 & 38 & 25 & 16 & 9 & 7 & 4\end{array}$

C

\section{D}

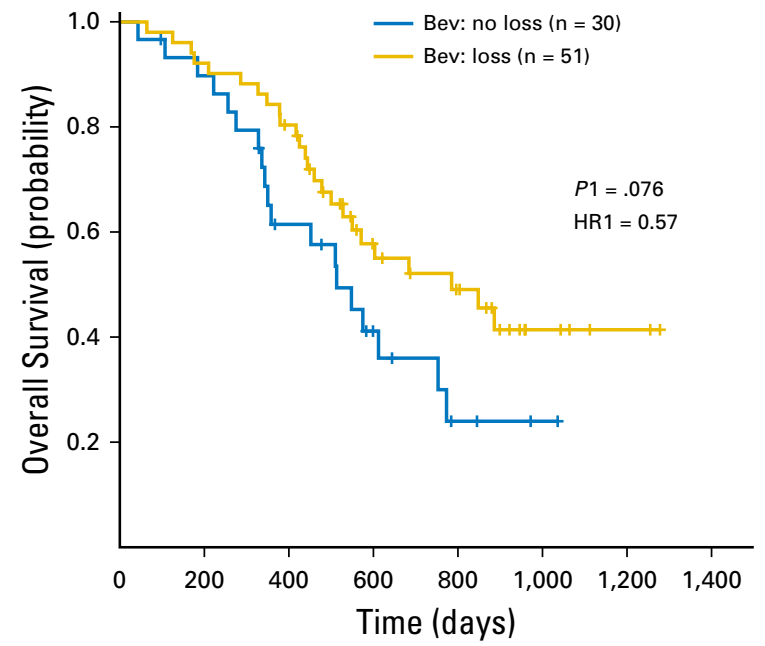

No. at risk

$\begin{array}{llllrrrrr}\text { Bev: no loss } & 30 & 26 & 16 & 8 & 3 & 1 & 0 & 0 \\ \text { Bev: loss } & 51 & 47 & 39 & 21 & 15 & 5 & 2 & 0\end{array}$

No. at risk

\begin{tabular}{lrrrrrrrr} 
Bev: no loss & 96 & 77 & 55 & 30 & 18 & 14 & 9 & 5 \\
Bev: loss & 214 & 198 & 152 & 105 & 73 & 42 & 27 & 16 \\
NoBev: no loss & 75 & 56 & 39 & 28 & 19 & 13 & 4 & 0 \\
NoBev: loss & 231 & 198 & 149 & 103 & 66 & 41 & 22 & 15 \\
\hline
\end{tabular}

Fig 3. Kaplan-Meier survival analysis for loss versus no loss of 18q11.2-q12.1 with respect to overall survival for all cohorts, and a meta-analysis of the combined data. (A)

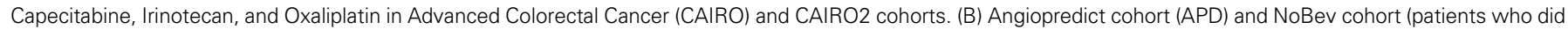

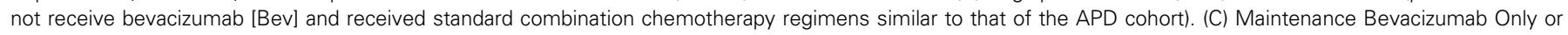

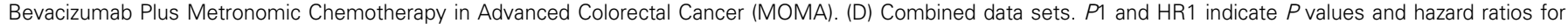
patients treated with Bev (blue lines $v$ gold lines). $P 2$ and HR2 indicate significance and hazard ratio for patients treated without Bev (red lines $v$ gray lines).

two potential confounding factors, MSI status and KRAS mutations. KRAS status is used as predictive marker for response to antiEGFR inhibition, which was given to a small minority of patients in the NoBev and APD cohorts $(n=3)$. Together, these data demonstrate that the loss of chromosome 18q11.2-q12.1 has independent predictive value for prolonged PFS in patients with mCRC treated with bevacizumab (Table 3).

\section{DISCUSSION}

A correlation between PFS and chromosome 18q11.2-q12.1 loss for patients with mCRC treated with bevacizumab, first reported by Haan et al, ${ }^{13}$ was validated in two large independent cohorts of patients treated with bevacizumab. Because the correlation of PFS 


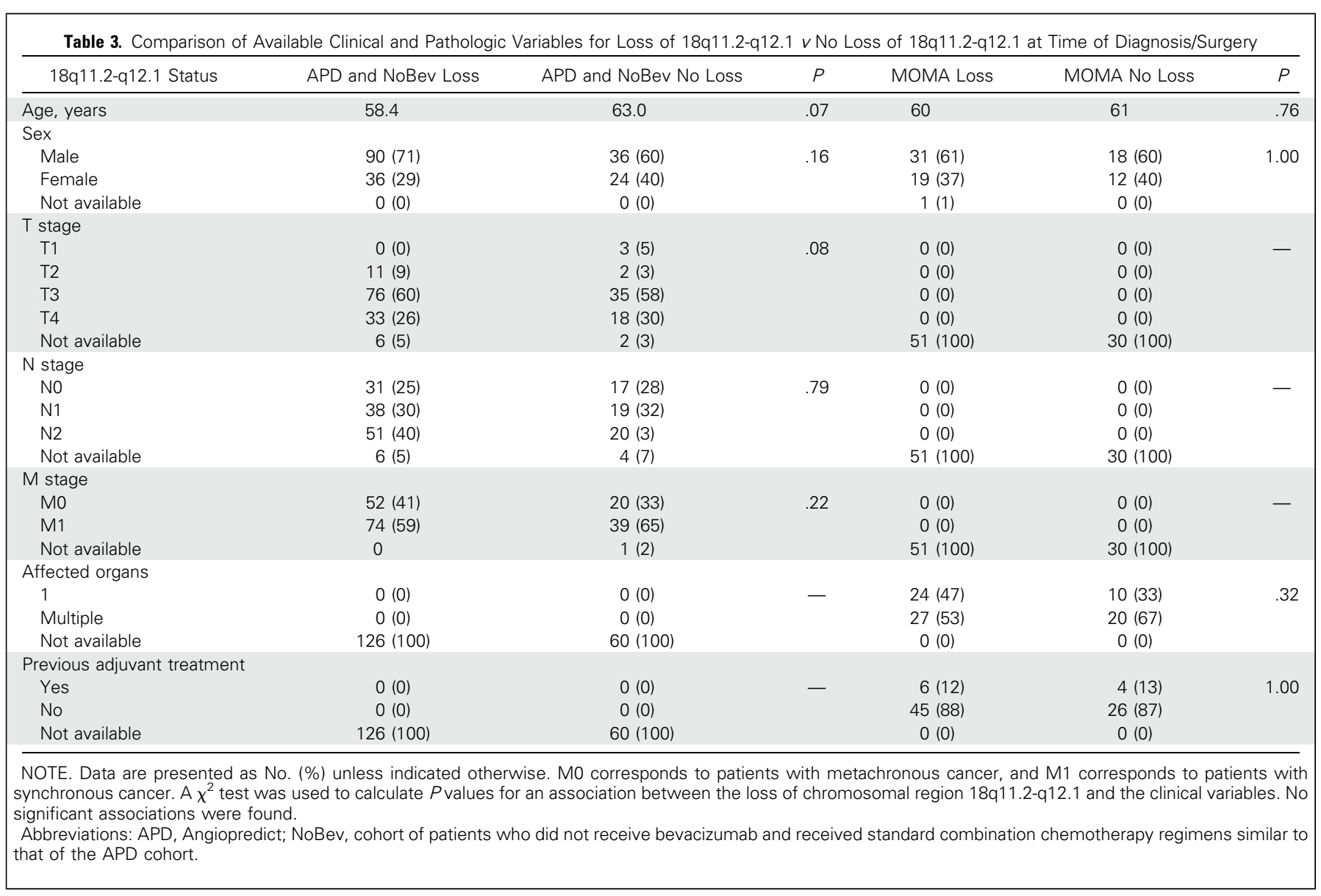

with 18q11.2-q12.1 loss was not observed in tumors of two large cohorts of patients with $\mathrm{mCRC}$ who received chemotherapy alone, we conclude that 18q11.2-q12.1 loss has predictive value for treatment with bevacizumab. The predictive value of this loss is supported by the statistical significance of an association with both OS and ORR in a combined analysis of all 310 patients treated with bevacizumab, which was not observed in the 306 patients treated with chemotherapy alone.

Loss of the entire chromosomal arm 18q has been observed previously to be a prognostic marker in several studies that considered non-mCRC primarily and hence, there was no bevacizumab-containing treatment. ${ }^{14-17}$ In a systematic review with a meta-analysis, it was concluded that although the loss of $18 \mathrm{q}$ is a poor prognostic indicator, results are variable among studies. ${ }^{31}$ Loss of the entire $18 \mathrm{q}$ arm was shown to correlate strongly with the vascularization of tumors in a cohort of 70 patients with nonmCRC. ${ }^{15}$ Hence, it is possible that the higher degree of vascular invasion in tumors with an $18 \mathrm{q}$ loss leads to a better response to the anti-angiogenic properties of bevacizumab. In our analysis, which considered only patients with mCRC, loss of the subcentromeric region 18q11.2-q12.1 had a slightly greater beneficial effect than loss of the entire chromosomal arm; in 91\% of these samples, the entire chromosome $18 \mathrm{q}$ arm was lost. The effects of bevacizumab treatment can therefore not be attributed necessarily to $18 \mathrm{q} 11.2-$ q12.1 alone; its effect may be multifactorial. There are 20 proteincoding genes located on 18q11.2-q12.1. Haan et $\mathrm{al}^{13}$ reported
LAM3 as the only gene significantly correlated with expression in colorectal cancer. LAM3 encodes for epiligrin, which is also expressed at wound sites of human skin. ${ }^{32} S M A D 4^{33}$ and $D C C^{34}$ are both located outside and proximal to the 18q11.2-q12.1 region. Both genes have been suggested as tumor suppressor genes; in addition, loss of SMAD4 has been linked to FU resistance ${ }^{35}$ and is mutated in $14 \%$ of mCRC tumors. ${ }^{36}$

Various approaches toward copy number analysis by shallow NGS have been introduced into the clinical diagnostic setting for formalin-fixed and paraffin-embedded solid tumor specimens. ${ }^{37}$ Shallow NGS copy number analysis may be combined cost effectively with NGS mutation analysis, ${ }^{25,30}$ necessary for KRAS and EGFR screening of samples from patients with $\mathrm{mCRC}^{18}$ in a clinical diagnostic setting.

The data presented here, which are supported by a robust and mature NGS copy number analysis platform, demonstrate that the loss of $18 \mathrm{q}$ should be considered in mCRC clinical decision making when considering supplementing combination chemotherapy with bevacizumab.

\section{AUTHORS' DISCLOSURES OF POTENTIAL CONFLICTS OF INTEREST}

Disclosures provided by the authors are available with this article at jco.org. 


\section{AUTHOR CONTRIBUTIONS}

Conception and design: Gerrit A. Meijer, Matthias P.A. Ebert, William M. Gallagher, Darran P. O'Connor, Diether Lambrechts, Annette T. Byrne, Nicole C.T. van Grieken, Bauke Ylstra

Financial support: Bauke Ylstra

Provision of study materials or patients: Cornelis J.A. Punt, Fotios Loupakis
Collection and assembly of data: Erik van Dijk, Hedde D. Biesma, Martijn Cordes, Dominiek Smeets, Maarten Neerincx, Sudipto Das, Paul P. Eijk, Verena Murphy, Anna Barat, Orna Bacon, Jochen H.M. Prehn, Johannes Betge, Timo Gaiser, Bozena Fender, Gerrit A. Meijer, Deborah A. McNamara, Rut Klinger, Miriam Koopman, Matthias P.A. Ebert, Elaine W. Kay, Bryan T. Hennesey, Henk M.W. Verheul, Darran P. O'Connor, Cornelis J.A. Punt, Fotios Loupakis, Nicole C.T. van Grieken, Bauke Ylstra Data analysis and interpretation: Erik van Dijk, Hedde D. Biesma, Martijn Cordes, Dominiek Smeets, Maarten Neerincx, Sudipto Das, Jochen H.M. Prehn, Cornelis J.A. Punt, Diether Lambrechts, Annette T. Byrne, Nicole C.T. van Grieken, Bauke Ylstra

Manuscript writing: All authors

Final approval of manuscript: All authors

Accountable for all aspects of the work: All authors

\section{REFERENCES}

1. Van Cutsem E, Cervantes $A$, Adam R, et al: ESMO consensus guidelines for the management of patients with metastatic colorectal cancer. Ann Oncol 27:1386-1422, 2016

2. Hurwitz $\mathrm{H}$, Fehrenbacher L, Novotny W, et al: Bevacizumab plus irinotecan, fluorouracil, and leucovorin for metastatic colorectal cancer. N Engl J Med 350:2335-2342, 2004

3. Saltz LB, Clarke S, Díaz-Rubio $E$, et al: Bevacizumab in combination with oxaliplatin-based chemotherapy as first-line therapy in metastatic colorectal cancer: A randomized phase III study. J Clin Oncol 26:2013-2019, 2008

4. Simkens LHJ, van Tinteren $H$, May A, et al: Maintenance treatment with capecitabine and bevacizumab in metastatic colorectal cancer (CAIRO3): A phase 3 randomised controlled trial of the Dutch Colorectal Cancer Group. Lancet 385:1843-1852, 2015

5. Franken MD, van Rooijen EM, May AM, et al: Cost-effectiveness of capecitabine and bevacizumab maintenance treatment after first-line induction treatment in metastatic colorectal cancer. Eur J Cancer 75:204-212, 2017

6. Goldstein DA, Chen $\mathrm{Q}$, Ayer T, et al: First- and second-line bevacizumab in addition to chemotherapy for metastatic colorectal cancer: A United Statesbased cost-effectiveness analysis. J Clin Oncol 33: 1112-1118, 2015

7. Messori A, De Rosa M, Fadda V, et al: Effectiveness and cost effectiveness of bevacizumab in metastatic colorectal cancer. J Clin Oncol 33: 3838-3839, 2015

8. Rosen LS, Jacobs IA, Burkes RL: Bevacizumab in colorectal cancer: Current role in treatment and the potential of biosimilars. Target Oncol 12:599-610, 2017

9. Hegde PS, Jubb AM, Chen D, et al: Predictive impact of circulating vascular endothelial growth factor in four phase III trials evaluating bevacizumab. Clin Cancer Res 19:929-937, 2013

10. Maru D, Venook AP, Ellis LM: Predictive biomarkers for bevacizumab: Are we there yet? Clin Cancer Res 19:2824-2827, 2013

11. Jubb AM, Hurwitz HI, Bai W, et al: Impact of vascular endothelial growth factor-A expression, thrombospondin-2 expression, and microvessel density on the treatment effect of bevacizumab in metastatic colorectal cancer. J Clin Oncol 24:217-227, 2006
12. Tol J, Koopman M, Cats A, et al: Chemotherapy, bevacizumab, and cetuximab in metastatic colorectal cancer. N Engl J Med 360:563-572, 2009

13. Haan JC, Labots M, Rausch C, et al: Genomic landscape of metastatic colorectal cancer. Nat Commun 5:5457, 2014

14. Jen J, Kim H, Piantadosi S, et al: Allelic loss of chromosome 18q and prognosis in colorectal cancer. N Engl J Med 331:213-221, 1994

15. Zhou W, Galizia G, Lieto E, et al: Counting alleles reveals a connection between chromosome $18 \mathrm{q}$ loss and vascular invasion. Nat Biotechnol 19:78-81, 2001 [Erratum: Nat Biotechnol 19:277, 2001]

16. Ogino S, Nosho K, Irahara N, et al: Prognostic significance and molecular associations of $18 q$ loss of heterozygosity: A cohort study of microsatellite stable colorectal cancers. J Clin Oncol 27:4591-4598, 2009

17. Cancer Genome Atlas Network: Comprehensive molecular characterization of human colon and rectal cancer. Nature 487:330-337, 2012

18. Koopman $\mathrm{M}$, Antonini $N F$, Douma J, et al: Sequential versus combination chemotherapy with capecitabine, irinotecan, and oxaliplatin in advanced colorectal cancer (CAIRO): A phase III randomised controlled trial. Lancet 370:135-142, 2007

19. Betge J, Barat A, Murphy V, et al: Outcome of colorectal cancer patients treated with combination bevacizumab therapy: A pooled retrospective analysis of three European cohorts from the Angiopredict Initiative. Digestion 94:129-137, 2016

20. Falcone A, Cremolini C, Loupakis F, et al: FOLFOXIRI plus bevacizumab (bev) followed by maintenance with bev alone or bev plus metronomic chemotherapy (metroCT) in metastatic colorectal cancer (mCRC): The phase II randomized MOMA trial. Ann Oncol 27:LBA21, 2016

21. Edge SB, Compton CC: The American Joint Committee on Cancer: The 7th edition of the AJCC cancer staging manual and the future of TNM. Ann Surg Oncol 17:1471-1474, 2010

22. Koopman M, Kortman GAM, Mekenkamp L, et al: Deficient mismatch repair system in patients with sporadic advanced colorectal cancer. $\mathrm{Br} J$ Cancer 100:266-273, 2009

23. Wang S, Zhang J, Lu W: Sample size calculation for the proportional hazards cure model. Stat Med 31:3959-3971, 2012

24. van de Wiel MA, Smeets SJ, Brakenhoff RH, et al: CGHMultiArray: Exact P-values for multi-array comparative genomic hybridization data. Bioinformatics 21:3193-3194, 2005
25. Scheinin I, Sie $D$, Bengtsson $H$, et al: DNA copy number analysis of fresh and formalin-fixed specimens by shallow whole-genome sequencing with identification and exclusion of problematic regions in the genome assembly. Genome Res 24: 2022-2032, 2014

26. Olshen $A B$, Venkatraman $E S$, Lucito $R$, et al: Circular binary segmentation for the analysis of arraybased DNA copy number data. Biostatistics 5: 557-572, 2004

27. van de Wiel MA, Brosens R, Eilers PHC, et al: Smoothing waves in array $\mathrm{CGH}$ tumor profiles. Bioinformatics 25:1099-1104, 2009

28. van de Wiel MA, Wieringen WN: CGHregions: Dimension reduction for array $\mathrm{CGH}$ data with minimal information loss. Cancer Inform 3:55-63, 2007

29. van de Wiel MA, Kim KI, Vosse SJ, et al: CGHcall: Calling aberrations for array CGH tumor profiles. Bioinformatics 23:892-894, 2007

30. Kuilman T, Velds A, Kemper K, et al: CopywriteR: DNA copy number detection from off-target sequence data. Genome Biol 16:49, 2015

31. Popat $S$, Houlston RS: A systematic review and meta-analysis of the relationship between chromosome 18q genotype, DCC status and colorectal cancer prognosis. Eur J Cancer 41:2060-2070, 2005

32. Ryan MC, Tizard R, VanDevanter DR, et al: Cloning of the LamA3 gene encoding the alpha 3 chain of the adhesive ligand epiligrin. Expression in wound repair. J Biol Chem 269:22779-22787, 1994

33. Jia X, Shanmugam C, Paluri RK, et al: Prognostic value of loss of heterozygosity and sub-cellular localization of SMAD4 varies with tumor stage in colorectal cancer. Oncotarget 8:20198-20212, 2017

34. Aschele C, Debernardis D, Lonardi S, et al: Deleted in colon cancer protein expression in colorectal cancer metastases: A major predictor of survival in patients with unresectable metastatic disease receiving palliative fluorouracil-based chemotherapy. J Clin Oncol 22:3758-3765, 2004

35. Zhang $B$, Zhang $B$, Chen $X$, et al: Loss of Smad4 in colorectal cancer induces resistance to 5-fluorouracil through activating Akt pathway. $\mathrm{Br} \mathrm{J}$ Cancer 110:946-957, 2014

36. Sarshekeh AM, Advani S, Overman MJ, et al: Association of SMAD4 mutation with patient demographics, tumor characteristics, and clinical outcomes in colorectal cancer. PLoS One 12:e0173345, 2017 [Erratum: PLoS One 12:e0178275, 2017]

37. Hastings RJ, Bown N, Tibiletti MG, et al: Guidelines for cytogenetic investigations in tumours. Eur J Hum Genet 24:6-13, 2016 


\section{Affiliations}

Erik van Dijk, Hedde D. Biesma, Martijn Cordes, Maarten Neerincx, Paul P. Eijk, Henk M.W. Verheul, Nicole C.T. van Grieken, and Bauke Ylstra, Vrije Universiteit Medical Center; Gerrit A. Meijer, Netherlands Cancer Institute; Cornelis J.A. Punt, Academic Medical Center, Amsterdam; Miriam Koopman, University Medical Center Utrecht, Utrecht, the Netherlands; Dominiek Smeets and Diether Lambrechts, KU Leuven, Leuven, Belgium; Sudipto Das, Orna Bacon, Jochen H.M. Prehn, Bryan T. Hennessey, Darran P. O'Connor, and Annette T. Byrne, Royal College of Surgeons in Ireland; Verena Murphy, Cancer Trials Ireland; Anna Barat, Deborah A. McNamara, and Elaine W. Kay, Beaumont Hospital; Bozena Fender and William M. Gallagher, OncoMark Ltd; Rut Klinger and William M. Gallagher, University College Dublin, Dublin, United Kingdom; Johannes Betge, Timo Gaiser, and Matthias P.A. Ebert, University Hospital Mannheim, Mannheim, Germany; and Fotios Loupakis, Veneto Institute of Oncology IOV-IRCCS Padua, Italy.

\section{Support}

Supported by the Seventh Framework Programme (Contract No. 278981 [ANGIOPREDICT]), the Dutch Cancer Society (Grant No. KWF 2015-7882), Science Foundation Ireland (Grant 13/CDA/2183 [COLOFORETELL] to A.T.B), and the State of Baden-Württemberg for Center of Geriatric Biology and Oncology (ZOBEL)-Perspektivförderung and Biology of Frailty-Sonderlinie Medizin (M.P.A.E.).

\section{Cancer.Net Provides Information for Your Spanish-Speaking Patients}

Find a variety of cancer information materials in Spanish at cancer.net/spanish or cancer.net/español for Spanishspeaking patients and their families and friends. 


\section{AUTHORS' DISCLOSURES OF POTENTIAL CONFLICTS OF INTEREST}

Loss of Chromosome 18q11.2-q12.1 Is Predictive for Survival in Patients With Metastatic Colorectal Cancer Treated With Bevacizumab

The following represents disclosure information provided by authors of this manuscript. All relationships are considered compensated. Relationships are self-held unless noted. I = Immediate Family Member, Inst = My Institution. Relationships may not relate to the subject matter of this manuscript. For more information about ASCO's conflict of interest policy, please refer to www.asco.org/rwc or ascopubs.org/jco/site/ifc.

\section{Erik van Dijk}

No relationship to disclose

Hedde D. Biesma

No relationship to disclose

\section{Martijn Cordes}

No relationship to disclose

Dominiek Smeets

No relationship to disclose

\section{Maarten Neerincx}

No relationship to disclose

\section{Sudipto Das}

Honoraria: Roche Sequencing

Paul P. Eijk

No relationship to disclose

Verena Murphy

No relationship to disclose

\section{Anna Barat}

No relationship to disclose

\section{Orna Bacon}

No relationship to disclose

Jochen H.M. Prehn

No relationship to disclose

\section{Johannes Betge}

Travel, Accommodations, Expenses: Celgene

\section{Timo Gaiser}

Consulting or Advisory Role: Roche/Genentech, Bristol-Myers Squibb, AstraZeneca, Merck Sharp \& Dohme

\section{Bozena Fender}

No relationship to disclose

\section{Gerrit A. Meijer}

Research Funding: Royal Philips (Inst), GlaxoSmithKline (Inst), Keosys SARL (Inst), Open Clinica (Inst), Roche Diagnostics Nederland B.V. (Inst), Exact Sciences (Inst), Genalice B.V. Harderwijk (Inst), The Hyve B.V. (Inst), OpenText (Inst), SURFsara B.V. (Inst), Vancis B.V. (Inst), CSC Computer Sciences B.V. (Inst), Sysmex (Inst), Sentinel CH. SpA (Inst) Patents, Royalties, Other Intellectual Property: Patents pending: protein biomarkers for detection of colorectal cancer in stool: NL 2008707, EP13720130.7, NL 2010276, US-2017-0199196 A1; combinations of protein biomarkers for detection of colorectal cancer in stool: EP 17172531.0 (Inst)

\section{Deborah A. McNamara}

No relationship to disclose

\section{Rut Klinger}

No relationship to disclose

\section{Miriam Koopman}

Consulting or Advisory Role: SERVIER

Research Funding: Bayer AG, Roche/Genentech, Merck Serono, SERVIER, Bristol-Myers Squibb

Matthias P.A. Ebert

Consulting or Advisory Role: Bristol-Myers Squibb Germany Speakers' Bureau: Shire

Research Funding: Bristol-Myers Squibb Germany

Elaine W. Kay

Consulting or Advisory Role: Almac Diagnostics

Bryan T. Hennesey

No relationship to disclose

Henk M.W. Verheul

Consulting or Advisory Role: Boehringer Ingelheim (Inst), Merck (Inst) Research Funding: Immunovo (Inst), Roche (Inst), Merck (Inst)

William M. Gallagher

Employment: OncoMark

Leadership: OncoMark

Stock or Other Ownership: OncoMark

Consulting or Advisory Role: Carrick Therapeutics

Research Funding: Carrick Therapeutics

Patents, Royalties, Other Intellectual Property: PCT/EP2015/071524: “A method of predicting risk of recurrence of cancer," licensed to OncoMark from University College Dublin and Trinity College Dublin; PCT/IE2008/ 000119: "Method and system for image analysis," licensed to OncoMark from University College Dublin

Travel, Accommodations, Expenses: OncoMark

Darran P. O'Connor

No relationship to disclose

Cornelis J.A. Punt

No relationship to disclose

Fotios Loupakis

Consulting or Advisory Role: Bayer AG, Genentech/Roche, Amgen Research Funding: Amgen

Diether Lambrechts

Speakers' Bureau: AstraZeneca

Research Funding: Biocartis NV (Inst), Multiplicom NV (Inst)

Patents, Royalties, Other Intellectual Property: WO 2013/030167: Responsiveness to angiogenesis inhibitors; WO 2013/030168 (EP2751280B1): Method for predicting risk of hypertension associated with anti-angiogenesis therapy; WO 2013/076029: Responsiveness to angiogenesis inhibitors; WO 2017/182656: Means and methods for anti-VEGF therapy (Inst)

Annette T. Byrne

No relationship to disclose

Nicole C.T. van Grieken

Consulting or Advisory Role: Bristol-Myers Squibb (Inst)

Bauke Ylstra

No relationship to disclose 
van Dijk et al

\section{Acknowledgment}

We thank Hendrik F. van Essen and Daoud Sie for their scientific support. The Dutch Colorectal Cancer Group is acknowledged for providing the CAIRO and CAIRO2 samples and clinical data. 